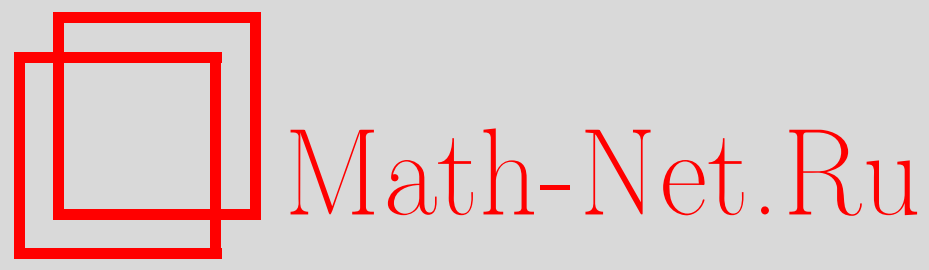

Ю. С. Самойленко, Л. Б. Туровская, О представлениях *-алгебр неограниченными операторами, Функи. анализ и его прил., 1997, том 31, выпуск 4, 80-83

DOI: https://doi.org/10.4213/faa497

Использование Общероссийского математического портала Math$\mathrm{Net.Ru}$ подразумевает, что вы прочитали и согласны с пользовательским соглашением

http://www.mathnet.ru/rus/agreement

Параметры загрузки:

IP : 18.207 .199 .55

26 апреля 2023 г., 13:25:43

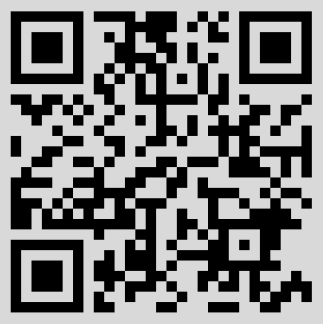




\section{О представлениях *-алгебр неограниченными операторами}

\section{(C) 1997. Ю. С. СамойлЕНКО, Л. Б. ТурОвСКАЯ}

В [1-3] изучается сложность структуры представлений алгебры $\mathfrak{A}$ над $\mathbb{C}$ с инволюцией * ограниченными операторами в комплексном гильбертовом пространстве $H$. Представления *-алгебры $\mathfrak{A}$, в частности, считаются сложными (алгебра $\mathfrak{A} p$-дикая), если они «содержат в качестве фрагмента» представления свободной *-алгебры $\mathfrak{B}$ с двумя самосопряженными образующими, т. е. существует *-гомоморфизм $\phi: \mathfrak{A} \rightarrow \mathfrak{B} \otimes M_{m}\left(M_{m}\right.$ - алгебра $m \times m$-матриц $)$, такой, что порожденный им функтор из категории представлений $\mathscr{R}(\mathfrak{B})$ в категорию представлений $\mathscr{R}(\mathfrak{A})$ является строгим и полным. Основания для того, чтобы считать представления такой алгебры сложными, получены в [1]: ее *-представления «содержат в качестве фрагмента» *-представления любой аффинной *-алгебры. Заметим, что если *-алгебра $p$-дикая, то она имеет факторпредставление, не являющееся представлением типа I. Обратное утверждение неверно (см. [2]).

В настоящей работе изучается сложность представлений коммутативной *-алгебры $\mathbb{C}\left[x_{1}, x_{2}\right]$ комплексных полиномов от вещественных переменных $x_{1}$, $x_{2}$ неограниченными операторами. Если представление $\pi$ такой алгебры интегрируемо, т.е. коммутируют спектральные проекторы замыканий операторов $\pi\left(x_{1}\right), \pi\left(x_{2}\right)$ или, что эквивалентно (см. [5]), существует плотное инвариантное относительно операторов представления линейное множество $\mathscr{D}$, состоящее из аналитических векторов для $\pi\left(x_{1}\right), \pi\left(x_{2}\right)$ и такое, что $\pi\left(x_{1}\right) \pi\left(x_{2}\right) \varphi=$ $\pi\left(x_{2}\right) \pi\left(x_{1}\right) \varphi$ для любых $\varphi \in \mathscr{D}$, то все неприводимые представления *-алгебры $\mathbb{C}\left[x_{1}, x_{2}\right]$ одномерные. В п. 2 работы доказано, что множество неинтегрируемых представлений устроено сложно и эти представления «содержат в качестве фрагмента» представления *-алгебры $\mathfrak{B}$. Для *-алгебры $\mathfrak{A}=\mathbb{C}\left\langle x_{1}, x_{2}\right|$ $\left.x_{i}^{*}=x_{i},\left[x_{1},\left[x_{1}, x_{2}\right]\right]=0\right\rangle$ в п. 3 показано, что сложно устроены даже представления $\pi$, определенные на плотной инвариантной относительно операторов представлений области, состоящей из аналитических векторов для операторов $\pi\left(x_{1}\right), \pi\left(x_{2}\right)$.

1. Приведем, в основном следуя [4], необходимые определения и результаты из теории представлений *-алгебр неограниченными операторами. Пусть $\mathfrak{A}$ есть *-алгебра, порожденная самосопряженными образующими $a_{1}, \ldots, a_{n}$ и единицей, которые удовлетворяют соотношениям

$$
P_{j}\left(a_{1}, \ldots, a_{n}\right)=0, \quad j=1, \ldots, m,
$$

где $P_{j}$ - полиномы от некоммутирующих переменных $a_{1}, \ldots, a_{n}$ над $\mathbb{C}$, и $\left(P_{j}(\cdot)\right)^{*}=P_{j}(\cdot)$.

* Работа первого автора частично поддержана грантом AMS, а второго - Международной Соросовской программой поддержки образования в отрасли точных наук (ISSEP), грант PSU061106, и грантом AMS. 
Рассмотрим самосопряженные представления $\pi$ *-алгебры $\mathfrak{A}$, такие, что операторы $\pi\left(a_{i}\right), i=1, \ldots, n$, существенно самосопряжены на области представления $\mathscr{D}(\pi)$ (см. [4]).

Оператор $T \in L(H, \widehat{H})$ будем называть сплетающим самосопряженные представления $\pi$ и $\hat{\pi}$, если $T E_{A_{i}}(\Delta)=E_{\widehat{A}_{i}}(\Delta) T$ для любых $\Delta \in \mathfrak{B}(\mathbb{R}), i=$ $1, \ldots, n$, где $A_{i}=\overline{\left.\pi\left(a_{i}\right)\right|_{\mathscr{D}(\pi)}}, \widehat{A}_{i}=\overline{\left.\hat{\pi}\left(a_{i}\right)\right|_{\mathscr{D}(\hat{\pi})}}$ и $E_{A}(\cdot)$ - разложение единицы самосопряженного оператора $A$. Множество таких операторов $T$ будем обозначать через $I(\pi, \hat{\pi})$. Согласно [4], $I(\pi, \hat{\pi})$ слабо замкнуто. Самосопряженные представления алгебры $\mathfrak{A}$ порождают $W^{*}$-категорию $\mathscr{R}(\mathfrak{A})$, объектами которой являются сами самосопряженные представления, а морфизмами - сплетающие операторы.

2. Пусть $\mathfrak{A}$ - коммутативная алгебра полиномов от вещественных переменных $x_{1}, x_{2}$. Вместо категории $\mathscr{R}(\mathfrak{A})$ рассмотрим эквивалентную категорию $\widetilde{\mathscr{R}}(\mathfrak{A})$, объектами которой являются пары унитарных операторов $(U, V)$, обратные преобразования Кэли которых порождают самосопряженное представление *-алгебры $\mathfrak{A}$ (т.е. существует область $\mathscr{D}(\pi) \subset \mathscr{D}\left(X_{1}\right) \cap \mathscr{D}\left(X_{2}\right)$, инвариантная относительно $X_{1}=i(U+I)(U-I)^{-1}, X_{2}=i(V+I)(V-I)^{-1}$ и такая, что операторы $\pi\left(x_{i}\right):=\left.X_{i}\right|_{\mathscr{D}(\pi)}, i=1,2$, определяют самосопряженное представление алгебры $\mathfrak{A}$, причем $\overline{\pi\left(x_{i}\right)}, i=1,2$, самосопряжены), а морфизмами ограниченные операторы, сплетающие разные пары $(U, V)$. Покажем, что представления $(U, V)$ «содержат в качестве фрагмента» *-представления алгебры $\mathfrak{B}$.

В основном следуя [4], рассмотрим следующую конструкцию. Пусть $a=a^{*}$, $b=b^{*}$ - образующие *-алгебры $\mathfrak{B}$, а $\rho$ - ее представление в пространстве $\mathscr{H}(\rho), \rho(a)=: A, \rho(b)=: B, A, B \in L(\mathscr{H}(\rho))$. В пространстве $W=\mathscr{H}(\rho) \oplus$ $\mathscr{H}(\rho) \oplus \mathscr{H}(\rho)$ определим пару операторов

$$
p=\left(\begin{array}{ccc}
\lambda I & \mu A & 0 \\
\mu A & \lambda I & \mu B \\
0 & \mu B & \lambda I
\end{array}\right), \quad q=\left(\begin{array}{ccc}
I & 0 & 0 \\
0 & 0 & 0 \\
0 & 0 & 0
\end{array}\right),
$$

где $\lambda, \mu \in \mathbb{R}$ таковы, что $1 / 2<p<3 / 4$. Кроме того, будем считать, что $A, B>$ 0 , в противном случае вместо $A, B$ следует взять операторы $A+(\|A\|+1) I, B+$ $(\|B\|+1) I$ соответственно. Положим $H=\bigoplus_{n \in \mathbb{Z}} H_{n}$, где $H_{n}=W \oplus W$. Любой вектор в $H$ может быть записан в виде $\left(\ldots, \varphi_{0}, \underline{\varphi_{1}}, \varphi_{2}, \ldots\right)$, где компонента с индексом 1 подчеркнута. В пространстве $H$ рассмотрим операторы

$$
\begin{aligned}
& v_{1}\left(\ldots, \varphi_{0}, \underline{\varphi_{1}}, \varphi_{2}, \ldots\right)=\left(\ldots, \underline{\varphi_{0}}, \varphi_{1}, \varphi_{2}, \ldots\right), \\
& v_{2}\left(\ldots, \varphi_{0}, \underline{\varphi_{1}}, \varphi_{2}, \ldots\right)=\left(\ldots, \underline{\varphi_{0}}, w_{1} \varphi_{1}, \varphi_{2}, \ldots\right), \\
& v_{3}\left(\ldots, \varphi_{0}, \underline{\varphi_{1}}, \varphi_{2}, \ldots\right)=\left(\ldots, \underline{\varphi_{0}}, w_{1} \varphi_{1}, w_{2} \varphi_{2}, \ldots\right),
\end{aligned}
$$

где $w_{i}: W \oplus W \rightarrow W \oplus W$,

$$
w_{1}=\left(\begin{array}{cc}
i(I-2 q) & 0 \\
0 & I
\end{array}\right), \quad w_{2}=\left(\begin{array}{cc}
I-2 p & -2\left(p-p^{2}\right)^{1 / 2} \\
-2\left(p-p^{2}\right)^{1 / 2} & 2 p-I
\end{array}\right) .
$$

Наконец, операторы $U, V$ определим в пространстве $\mathscr{H}(\pi)=\bigoplus_{n \in \mathbb{Z}} \mathscr{H}_{n}$, где 
$\mathscr{H}_{n}=H$, следуюшим образом:

$$
\begin{aligned}
& U\left(\ldots, \varphi_{0}, \underline{\varphi_{1}}, \varphi_{2}, \ldots\right)=\left(\ldots, \underline{\varphi_{0}}, \varphi_{1}, \varphi_{2}, \ldots\right), \\
& V\left(\ldots, \varphi_{0}, \underline{\varphi_{1}}, \varphi_{2}, \ldots\right)=\left(\ldots, v_{0} \varphi_{0}, \underline{v_{1} \varphi_{1}}, v_{2} \varphi_{2}, \ldots\right),
\end{aligned}
$$

где $v_{n}=v_{1}$ при $n<1$ и $v_{n}=v_{3}$ при $n>3$. Подобно тому, как это делается в [4], доказывается следующее

ПРЕДЛОЖЕНИЕ 1. Операторь $(U, V)$ порождают самосопряженное представление $\pi$ *-аллебрь $\mathbb{C}\left[x_{1}, x_{2}\right]$ на $\mathscr{D}(\pi)=\mathscr{D}_{\infty}\left(X_{1}, X_{2}\right)$, где $\mathscr{D}_{\infty}\left(X_{1}, X_{2}\right)=$ $\left\{\varphi \in \mathscr{H}(\pi) \mid \varphi \in \mathscr{D}\left(X_{1}^{n} X_{2}^{m}\right) \cap \mathscr{D}\left(X_{2}^{m} X_{1}^{n}\right)\right.$ u $\left.X_{1}^{n} X_{2}^{m} \varphi=X_{2}^{m} X_{1}^{n} \varphi, n, m \in \mathbb{N}\right\}$.

ЗАмечаниЕ 1 . Операторы $X_{i}^{n}, i=1,2$, самосопряжены в существенном на $\mathscr{D}(\pi)$ для любого $n \in \mathbb{N}$.

Функтор $F$ из категории $\mathscr{R}(\mathfrak{B})$ в категорию $\widetilde{\mathscr{R}}(\mathfrak{A})$ определим следующим образом:

- если $\rho$ - представление алгебры $\mathfrak{B}, \rho(a)=A, \rho(b)=B, A, B \in L(\mathscr{H}(\rho))$, то $F(\rho)=(U, V)$,

- если $C: \mathscr{H}(\rho) \rightarrow \mathscr{H}(\hat{\rho}), C A=\widehat{A} C, C B=\widehat{B} C$, то $F(C)=\mathscr{C}=\operatorname{diag}(\ldots$, $C, C, \ldots)$ и $\mathscr{C}: \mathscr{H}(\pi) \rightarrow \mathscr{H}(\hat{\pi})$.

Теорема 1. Функтор $F$ строгий и полный.

3. Рассмотрим $*$-алгебру $\mathfrak{A}=\left\langle x_{1}, x_{2} \mid x_{i}^{*}=x_{i},\left[x_{1},\left[x_{1}, x_{2}\right]\right]=0\right\rangle$. Очевидно, что любое самосопряженное представление коммутативной алгебры $\mathbb{C}\left[x_{1}, x_{2}\right]$ является самосопряженным представлением *-алгебры $\mathfrak{A}$. Как следует из предыдущего пункта, такие представления могут быть устроены сложно. В данном пункте мы покажем, что, в отличие от алгебры $\mathbb{C}\left[x_{1}, x_{2}\right]$, сложно устроены уже представления $\pi$, определенные на плотной инвариантной относительно операторов представления области $\mathscr{D}$, состоящей из аналитических векторов для операторов $\pi\left(x_{1}\right), \pi\left(x_{2}\right)$.

Следуя [6], рассмотрим следующую конструкцию. Пусть $\rho$ - представление *-алгебры $\mathfrak{B}$ в сепарабельном гильбертовом пространстве $\mathscr{H}(\rho), \rho(a)=: A$, $\rho(b)=: B, A, B \in L(\mathscr{H}(\rho))$. В пространстве $H(\pi)=(\mathscr{H}(\rho) \oplus \mathscr{H}(\rho) \oplus \mathscr{H}(\rho)) \otimes$ $L_{2}(\mathbb{R}, d x)$ рассмотрим максимальные операторы $X_{1}=I_{\mathscr{H}}(\rho)^{3} \otimes M_{x}, X_{2}=A_{1} \otimes$ $i d / d x+A_{2} \otimes I_{L_{2}(\mathbb{R}, d x)}$, где $M_{x}$ - оператор умножения на $x, I_{H}$ - единичный оператор в пространстве $H$,

$$
A_{1}=\left(\begin{array}{ccc}
\lambda_{1} I_{\mathscr{H}(\rho)} & 0 & 0 \\
0 & \lambda_{2} I_{\mathscr{H}(\rho)} & 0 \\
0 & 0 & \lambda_{3} I_{\mathscr{H}(\rho)}
\end{array}\right), \quad A_{2}=\left(\begin{array}{ccc}
0 & A & B \\
A & 0 & 0 \\
B & 0 & 0
\end{array}\right),
$$

$\lambda_{i} \in \mathbb{R}, \lambda_{i} \neq \lambda_{j}, i \neq j$. Кроме того, будем считать, что $A, B>0$. Обозначим через $H^{w}(A)$ множество аналитических векторов для оператора $A$. Известно, что существует плотное линейное множество $\Phi \subset L_{2}(\mathbb{R}, d x)$, такое, что $\Phi$ инвариантно относительно операторов $M_{x}, i d / d x$ и $\Phi \subset H^{w}\left(M_{x}\right) \cap H^{w}(d / d x)$. Положим $\mathscr{D}(\pi)=$ л. о. $\{g \otimes h \mid g \in \mathscr{H}(\rho) \oplus \mathscr{H}(\rho) \oplus \mathscr{H}(\rho), h \in \Phi\}, \pi\left(x_{1}\right)=\left.X_{1}\right|_{\mathscr{D}(\pi)}$, $\pi\left(x_{2}\right)=\left.X_{2}\right|_{\mathscr{D}(\pi)}$. Очевидно, что $\mathscr{D}(\pi)$ плотно в $H(\pi)$ и инвариантно относительно $X_{i}, i=1,2$. Пусть $\mathscr{D}\left(\pi^{*}\right)=\bigcap_{r \in \mathbb{N}} \bigcap_{\left(j_{1}, \ldots, j_{r}\right) \in J^{r}} \mathscr{D}\left(\pi\left(x_{j_{r}}\right)^{*} \cdots \pi\left(x_{j_{1}}\right)^{*}\right)$, $J=\{1,2\}$. 
ПРЕДЛОЖЕНИЕ 2. (а) $\mathscr{D}(\pi) \subset H^{w}\left(X_{1}\right) \cap H^{w}\left(X_{2}\right)$;

(б) операторь $X_{1}, X_{2}$ определяют самосопряженное представление на $\mathscr{D}\left(\pi^{*}\right) \supset \mathscr{D}(\pi)$.

Определим функтор $F$ из категории $\mathscr{R}(\mathfrak{B})$ в категорию $\mathscr{R}(\mathfrak{A})$ следующим образом:

- если $\rho$ - представление *-алгебры $\mathfrak{B}, \rho(a)=A, \rho(b)=B$, то $F(\rho)=\pi^{*}$ $\left(\pi^{*}\right.$ - представление *-алгебры $\mathfrak{A}$ на $\mathscr{D}\left(\pi^{*}\right)$, порожденное операторами $\left.\left.X_{i}\right|_{\mathscr{D}\left(\pi^{*}\right)}, i=1,2\right)$;

- если $C: \mathscr{H}(\rho) \rightarrow \mathscr{H}(\hat{\rho}), C A=\widehat{A} C, C B=\widehat{B} C$, то

$$
F(C)=\mathscr{C}=\left(\begin{array}{ccc}
C & 0 & 0 \\
0 & C & 0 \\
0 & 0 & C
\end{array}\right) \otimes I_{L_{2}(\mathbb{R}, d x)} .
$$

ТЕОРЕМА 2. Функтор $F$ строгий и полньй.

\title{
ЛИТЕРАТУРА
}

1. Кругляк С. А., Самойленко Ю. С. Функц. анализ и его прил., 14, вып. 1, 60-62 (1980). 2. Пирятинская А. Ю., Самойленко Ю. С. Укр. матем. ж., 47, № 1, 70-78 (1995). 3. Kruglyak S., Piryatinskaya A. Preprint, Institut Mittag-Leffler (1996). 4. Schmüdgen $K$. Unbounded operator algebras and representation theory, AkademieVerlag, Berlin, 1990. 5. Nelson E. Ann. Math. 70, No. 3, 572-615 (1959). 6. Nizhnik L. P., Turowska L. B. Methods of Func. Anal. and Topol. В печати.

Институт математики НАН Украины, Киев

Поступило в редакцию 11 сентября 1996 г.

УДК 517.9

\section{Об условиях конечности точечного спектра в несамосопряженной модели Фридрихса ${ }^{\star}$}

\author{
(C) 1997. С. А. Степин
}

В работе изучается точечный спектр $\sigma_{p}$ операторов в $L_{2}(l), l=[a, b] \subset \mathbb{R}$, вида $H=H_{0}+V$, где $H_{0}$ - оператор умножения на независимую переменную, а возмущение $V$ - интегральный оператор с ядром $v$, удовлетворяющим некоторым условиям гладкости.

Известно (см. [1-3]), что спектральные свойства операторов самосопряженной модели Фридрихса существенно зависят от степени гёльдеровской гладкости ядра возмущения. В этих терминах гранищей конечности сингулярного спектра служит показатель гёльдеровости $1 / 2$. В несамосопряженном случае даже при условии бесконечной дифференцируемости ядра $v$ может происходить сгущение собственных значений к отрезку $l$; в [4] построен соответствующий пример и исследована структура множества точек накопления собственных значений.

\footnotetext{
* Работа выполнена при поддержке РФФИ (грант № 96-01-00333).
} 